\title{
PHOTOCHEMICAL CHARACTERISTICS OF DICLOFENAC AND ITS PHOTODEGRADATION OF INCLUSION COMPLEXES WITH $\beta$-CYCLODEXTRINS
}

\author{
Minmin Qin and Haifeng Yang \\ School of Biology and Environmental Science, Shanghai Normal University, ShangHai 200234, PR China \\ Shengwen Chen*, Hongyong Xie and Jie Guan \\ School of Urban Development and Environment Engineering, Shanghai Second Polytechnic University, ShangHai 201209, PR China
}

Recebido em 3/7/11; aceito em 29/9/11; publicado na web em 6/12/11

\begin{abstract}
Diclofenac is one of most frequently detected compounds in the water cycle. In this work, the effect of initial concentration, liquid inclusion complexes with $\beta$-Cyclodextrins ( $\beta$-CDs) on the photodegradation of diclofenac were studied. Six phototransformation products were detected by HPLC chromatograms. UV-absorption spectra of diclofenac and phototransformation products were determined. One of the phototransformation products was identified. The degradation followed pseudo-first-order kinetics. The experiment showed that irradiation of diclofenac in the presence of $\beta$-CDs increase photodegradation rate and determined the optimal molar ratio of diclofenac to $\beta$-CDs as 1:2. The reduced photohaemolytic activity of diclofenac in the presence of $\beta$-CDs may be attributed to the sequestering and stabilizing of the radical intermediates and /or photoproducts by complexation.
\end{abstract}

Keywords: diclofenac; $\beta$-cyclodextrins; photodegradation.

\section{INTRODUCTION}

Diclofenac (2-[2',6'-(dichlorophenyl) amino] phenyl acetic acid, DCF), a popular non-steroidal anti-inflammatory drug, is used to treat inflammatory and painful diseases. ${ }^{1}$ Nowadays, this drug is ubiquitously present in the aquatic environment. ${ }^{2}$ These contaminants are important because many of them are not degraded by the typical biological treatments applied in wastewater treatment plants and represent a continuous input into the environment. ${ }^{3}$ Within the aquatic environment, in river and surface waters, diclofenac is one of the most frequently detected pharmaceuticals. ${ }^{4}$

Diclofenac is considerably stable under normal environmental conditions and the most probable decomposition pathway for its onsite elimination is photodecomposition. ${ }^{5-8}$ Investigation of the photolysis of labile environmental pharmaceutical contaminants is important in environmental chemistry because it presents basic data on their fates and persistence in natural environments exposed to sunlight action. ${ }^{9}$

Cyclodextrins (CDs) are cyclic oligosaccharides made up of six to eight $\alpha$-D-glucose units connected through glycosidic- $\alpha$-1,4-bonds . They are composed of a hydrophobic internal cavity and hydrophilic external surface. ${ }^{10}$ Thus, they have the ability to form an inclusion complex with various guest molecules of suitable polarity and dimension. ${ }^{11-13}$ This kind of special molecular structure allows them to form host/guest inclusion complexes with various guest molecules that have suitable polarity and dimension, ${ }^{14}$ which can be characterized qualitatively and quantitatively by fluorescence due to the considerable alteration in photophysical properties. ${ }^{15}$ It has been reported that diclofenac forms water-soluble complexes with $\beta$-CDs. ${ }^{16-18}$

Kitano et al. reported that bisphenols, such as bisphenol A, are able to form an inclusion complex with CDs and its derivatives ${ }^{19}$ and their potential as media for controlling chemical and photochemical reactions has been extensively proven. ${ }^{20}$ In environmental research, because CDs are nonpoisonous and biodegradable, they have been used to promote degradation of non-biodegradable pollutants discharged into aqueous environments. Kamiya et al. ${ }^{13}$ investigated

*e-mail: swchen@eed.sspu.cn that the inclusion effect of $\beta$-CDs on the photodegradation rate of Paraoxon and found that $\beta$-CDs had a substantial promoting effect. $^{14,21}$

In this work, the formation of an inclusion complex between diclofenac and $\beta$-CDs was studied and the enhanced photodegradation behavior of diclofenac in the presence of $\beta$-CDs was investigated under different light irradiation. Effects of initial concentration and the types of light on the photodegradation of diclofenac as well as reaction kinetics were discussed.

\section{EXPERIMENTAL}

\section{Reagents}

Diclofenac sodium salt was purchased from Sichuan XiYa chemical Co. Ltd. $\beta$-CDs were purchased from Sinopharm Chemical Reagent Co. Ltd. Methanol was of HPLC grade (Sinopharm Chemical Reagent Co.). Hydrochloric acid and $\mathrm{NaOH}$ were used to adjust the $\mathrm{pH}$ values of the solutions. All other reagents were of analytic reagent grade. Deionized water was used in all experiments.

\section{Irradiation procedures}

The photocatalytic degradation of diclofenac was conducted on an XPA-7 type photochemical reactor (Xujiang Machine Factory, Nanjing, China) (Figure 1) equipped with a $100 \mathrm{~W}$ mercury lamp (mean wavelength $365 \mathrm{~nm}$ ) with light intensities at quartz tube positions of $12.7 \mathrm{~mW} / \mathrm{cm}^{2}$ (measured using a UV-A irradiation meter, Beijing Normal University, China). A $100 \mathrm{~W}$ halogen lamp and a $100 \mathrm{~W}$ Xenon lamp were also used as light sources separately. The temperatures of the reaction solutions were maintained at $25 \pm 1{ }^{\circ} \mathrm{C}$ by cooling water circulation.

Degradations were performed on $50 \mathrm{~mL}$ of aqueous solutions containing the different initial concentrations of diclofenac, according to the preliminary test. Aliquots of $2 \mathrm{~mL}$ suspension were sampled using a syringe at specific time intervals and filtrated through a 0.45 $\mu \mathrm{m}$ polymer membrane filters in a syringe-end filter. The $\mathrm{pH}$ value of the filtrates was determined immediately after filtration. 


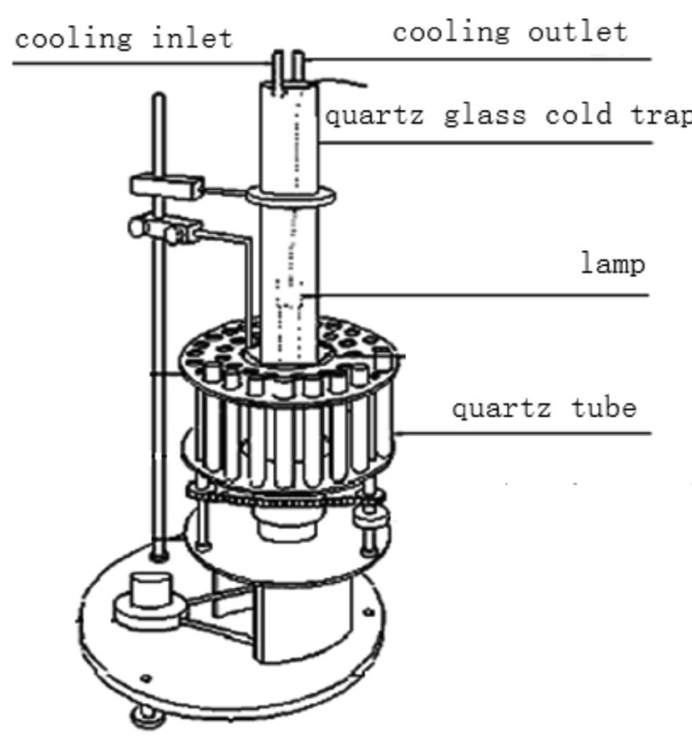

Figure 1. Schematic diagram of photochemical reactor

\section{Analysis}

\section{HPLC-analysis}

Diclofenac in aqueous solution was measured by HPLC (Shimadzu LC-20A pump, Shim-pack VP-DDS-C18 column $(4.6 \times 150 \mathrm{~mm}, 3 \mu \mathrm{m}))$ at a flow rate of $0.8 \mathrm{~mL} \mathrm{~min}^{-1}$ and using a UV detector (SPD-20A detector) at $245 \mathrm{~nm}$. The injection volume was $10 \mu \mathrm{L}$. The eluent comprised $60 \%$ methanol and $40 \%$ deionized water $(\mathrm{pH}=2.5)$. Diclofenac was determined by the retention time of $12.5 \mathrm{~min}$ and its concentration was obtained by the peak area.

\section{RESULTS AND DISCUSSION}

\section{Results of HPLC chromatogram analysis and photo transformations}

In this study, products of photochemical reactions were identified by retention times (External reference method) on HPLC. Typical HPLC chromatograms different irradiation times under a $100 \mathrm{~W}$ mercury lamp are shown in Figure 2. Results show that only diclofenac at the retention time $12.5 \mathrm{~min}$ was detected before irradiation, and that the peak height decreased with increasing irradiation time, almost disappearing after $18 \mathrm{~min}$ of irradiation. The concentration of diclofenac in the experiments was obtained from the peak area at the retention time of $12.5 \mathrm{~min}$. There was a peak (peak 6) at the retention time $9.5 \mathrm{~min}$ and the peak value first increased, and then decreased, with increasing irradiation time. After $18 \mathrm{~min}$ of irradiation, the peak at retention time 9.5 min disappeared. Five peaks appeared at the retention time from 3 to $5 \mathrm{~min}$ and the peak areas increased with increasing irradiation time. As shown in Figure 2, six phototransformation products were formed during irradiation with a $100 \mathrm{~W}$ high pressure mercury lamp and other light sources.

The absorption spectrum of diclofenac and phototransformation in aqueous solution observed are shown in Figure 3. Comparing with the literature, ${ }^{22}$ the UV spectrum of peak 6 was the same as that of $\mathrm{Cz} 1$ ((8-chloro-9H-carbazol-1-yl) acetic acid) (Figure 3S, supplementary material ). The $\mathrm{Cz} 1$ also increased at first and then decreased with increasing irradiation time. Drawing on the above points, peak 6 must be (8-chloro-9H-carbazol-1-yl) acetic acid. Peak 6 ((8-chloro-9H-carbazol-1-yl) acetic acid) was the primary transformation product of diclofenac under the conditions studied.

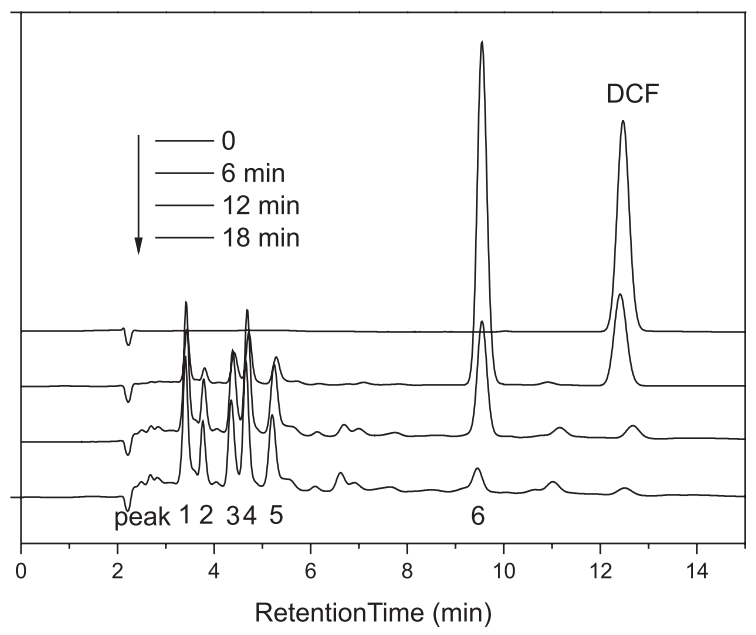

Figure 2. HPLC chromatograms of diclofenac photodegradation under 100 W mercury lamp under different irradiation time

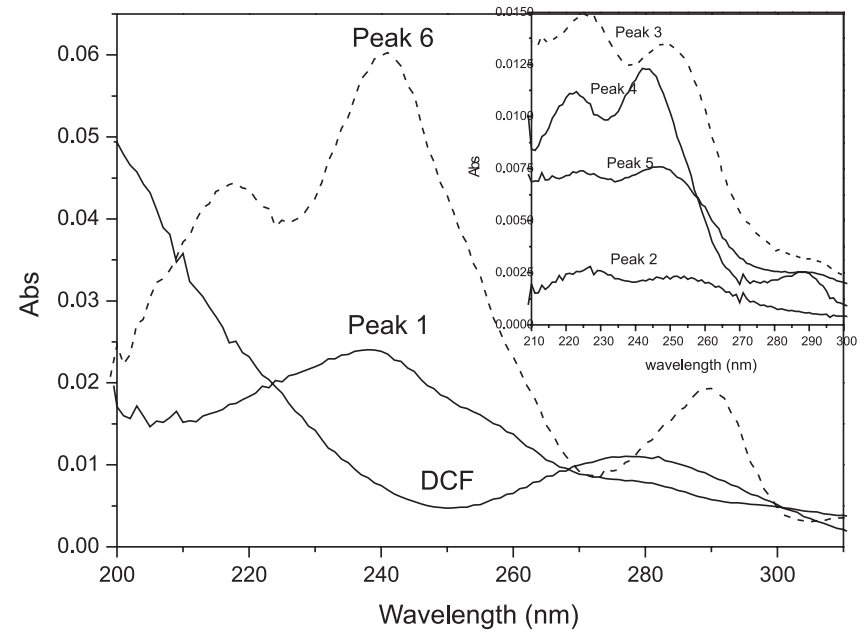

Figure 3. Absorption spectra of diclofenac and phototransformation products

\section{Photodegradation kinetics of diclofenac and pH change}

Experimental results indicate that the destruction rates of organic contaminants can be described by Equation 1:

$$
R=-\frac{d C}{d t}=k_{a p p} C_{0}
$$

The photo-inducted treatment started at neutral $\mathrm{pH}$ (the $\mathrm{pH}$ produced by dissolving diclofenac in deionized water) but varied during the experiment from 7.35 to 4.20, as shown in Figure 4, due to dechlorination of the diclofenac molecule and formation of carboxylic acids.

Diclofenac concentration versus irradiation time at different initial concentrations is plotted in Figure 4. On Figure 4, the vertical axis is $C / C_{0}$ to illustrate overall pseudo-first-order kinetics $\left(\operatorname{lnC}_{0} / C=k_{a p p} t\right)$ in the case of initial concentration less than $40 \mathrm{mg} \mathrm{L}^{-1}$. The first order rate constant $k_{a p p}$ is obtained from the slop of concentration versus irradiation time curve. The initial rate $\left(R=k_{a p p} C_{0}\right)$ can thus be obtained. The experiments were carried out from a range of initial concentrations 5.0, 10.0, 20.0, and $40.0 \mathrm{mg} \mathrm{L}^{-1}$. The results are shown in Figure 5. It is evident that the initial rate of diclofenac photodegradation rises with increasing diclofenac concentration. The apparent rate constant $k_{a p p}$ of diclofenac decreased from 0.43 
to $0.23 \mathrm{~min}^{-1}$ with increasing diclofenac concentration of 5 to $40 \mathrm{mg} \mathrm{L}^{-1}$.

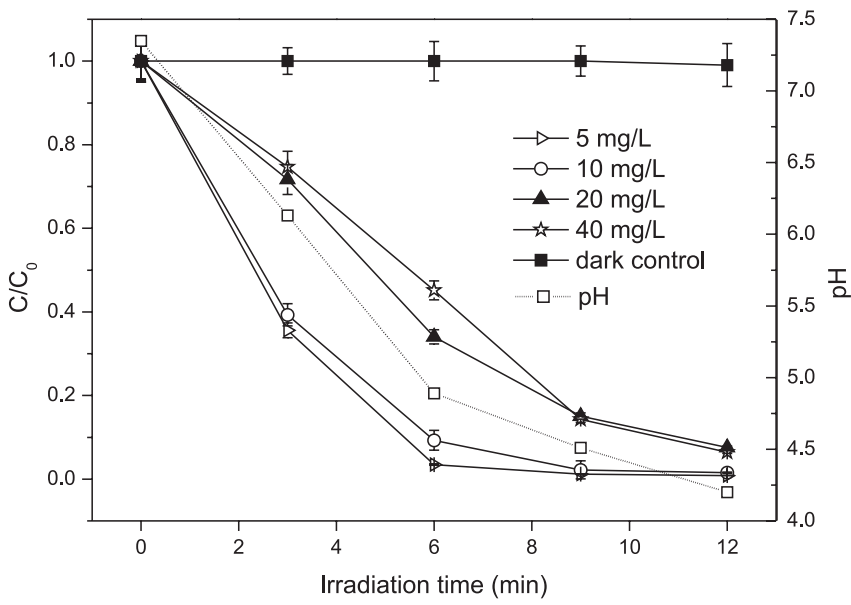

Figure 4. $\mathrm{C} / \mathrm{C}_{0}$ and $\mathrm{pH}$ versus irradiation time

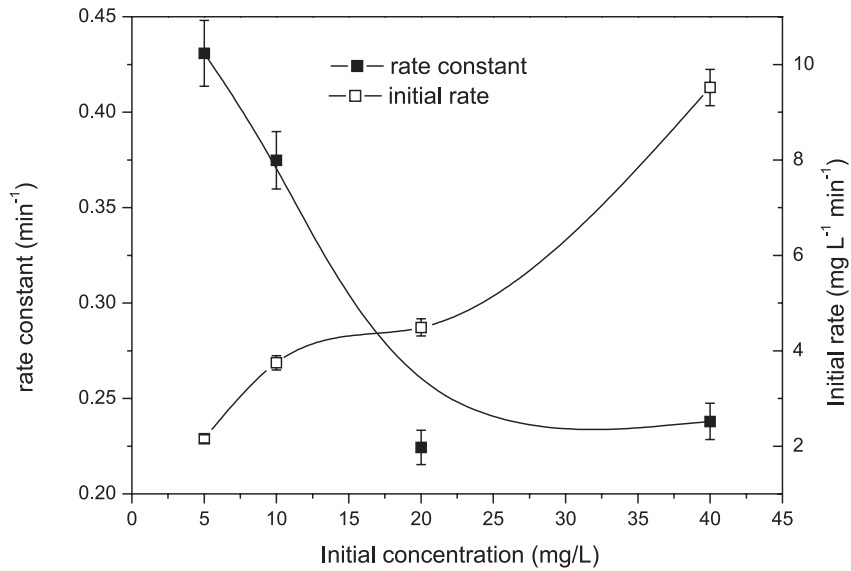

Figure 5. The rate constant and the initial rate constant of different initial concentrations

\section{Photodegradation under different light sources}

Experiments were performed at an initial concentration of $40 \mathrm{mg} \mathrm{L}^{-1}$ under the irradiation of four types of light source, namely, high pressure mercury lamp $100 \mathrm{~W}$, Halide Lamp $100 \mathrm{~W}$, Xenon lamp $100 \mathrm{~W}$ and sunlight (sunny day, January, 2010; location $121.64897^{\circ} \mathrm{E}$, $31.26359^{\circ} \mathrm{N}$ ). The plot of Diclofenac concentration versus irradiation time is shown in Figure 6. Degradation of diclofenac was faster under high pressure mercury lamp than other conditions. The photodegradation reaction obeyed overall pseudo-first-order kinetics under the different kinds of light source. The rate constants $k_{a p p}$ obtained from the slope of the diclofenac concentration versus irradiation time curve are listed in Table 1. From Table 1, it can be seen that the rate of photodegradation under the different kinds of light source in descending order ofmagnitude, was Mercury lamp $>$ Halide lamp $>$ Xenon lamp $>$ Sunlight.

\section{Photodegradation of diclofenac with CDs}

Diclofenac solution with and without $\beta$-CDs, was irradiated under a $100 \mathrm{~W}$ Mercury lamp light giving the results shown in Figure 7. It has been reported that ketoprofen form water-soluble complexes with $\beta$-CDs that are less active than the non-complexed compounds in photohaemolysis. Kinetic studies have revealed that the presence of Hydroxypropy- $\beta$-cyclodextrin reduced drug

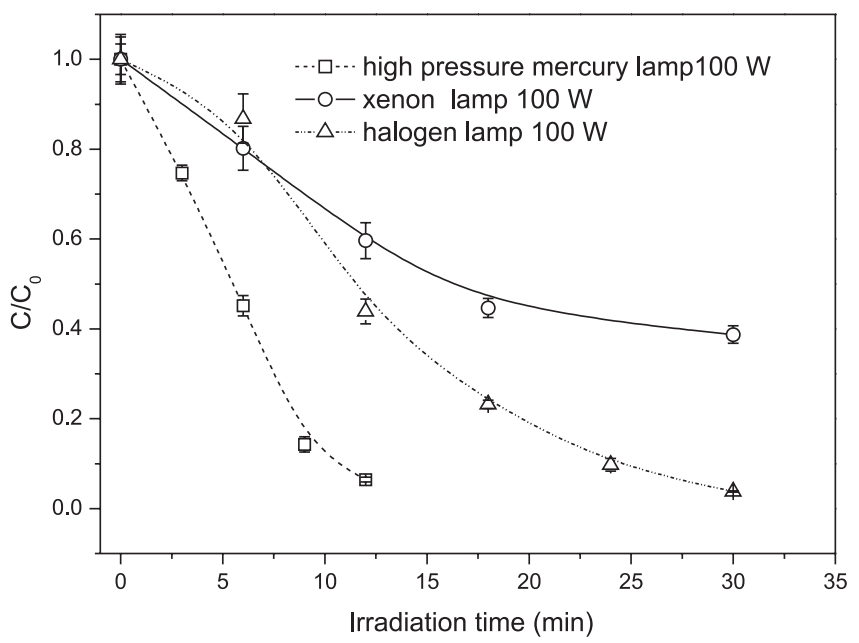

Figure 6. $C / C_{0}$ irradiation under the different light sources of $40 \mathrm{mg} \mathrm{L}^{-1}$ of diclofenac

Table 1. The rate constant $k_{a p p}$ and initial rate $R$ under the different light sources irradiation of $40 \mathrm{mg} \mathrm{L}^{-1}$ of diclofenac

\begin{tabular}{lccc}
\hline Light sources & $k_{a p p}\left(\mathrm{~min}^{-1}\right)$ & $R\left(\mathrm{mg} \mathrm{L}^{-1} \mathrm{~min}^{-1}\right)$ & $t_{1 / 2}(\mathrm{~min})$ \\
\hline Sun light & 0.00613 & 0.245 & 113 \\
100 W Xenon lamp & 0.0465 & 1.86 & 14.9 \\
100 W Halogen Lamp & 0.112 & 4.48 & 6.19 \\
100 W Mercury lamp & 0.238 & 9.51 & 2.91 \\
\hline
\end{tabular}

naproxen photogradation. ${ }^{23}$ However, Figure 7 shows that formation of inclusion complexes of DCF with $\beta$-CDs was proven to increase diclofenac photodegradation. The results are accordance with naproxen with $\beta$-CDs. Jimenez reported that irradiation of naproxen in the presence of $\beta$-CDs leads to more rapid disappearance of the drug naproxen. ${ }^{24}$ It appears that photohaemolytic activity of diclofenac is reduced in the presence of $\beta$-CDs. This can be attributed to the different behavior of the complexed radical intermediates, rather than to greater drug photostability.

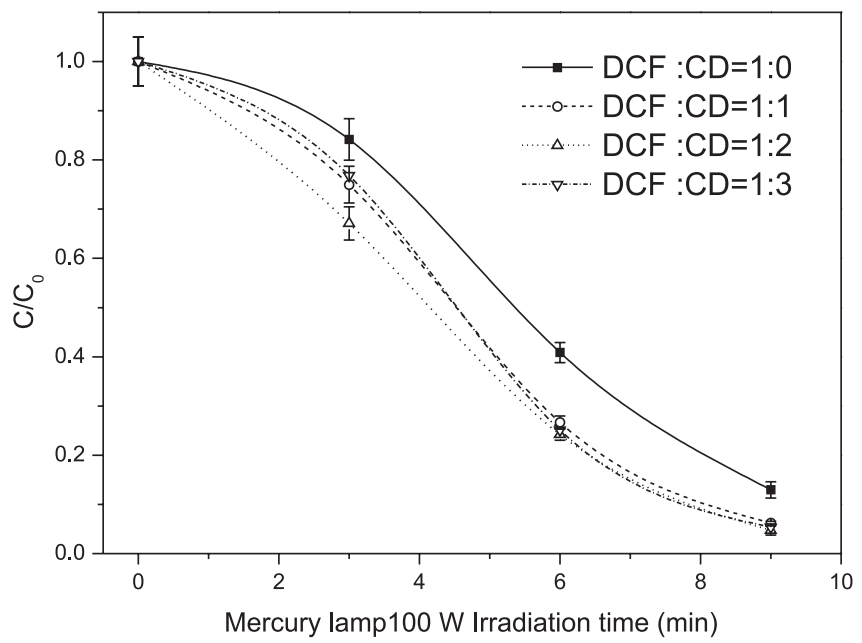

Figure 7. Effect of mole ratio of diclofenac to $\beta-C D$ under 100 W Mercury lamp

The photodegradation reaction obeyed overall pseudo-first-order kinetics. The rate constant $k_{a p p}$ versus the ratio of diclofenac to $\beta$-CDs under $100 \mathrm{~W}$ Mercury lamp light is plotted in Figure 8. The results suggest that $\beta$-CDs induce enhancement of diclofenac 
photodegradation and that $1: 2$ is the optimum molar ratio of diclofenac to $\beta$-CDs.

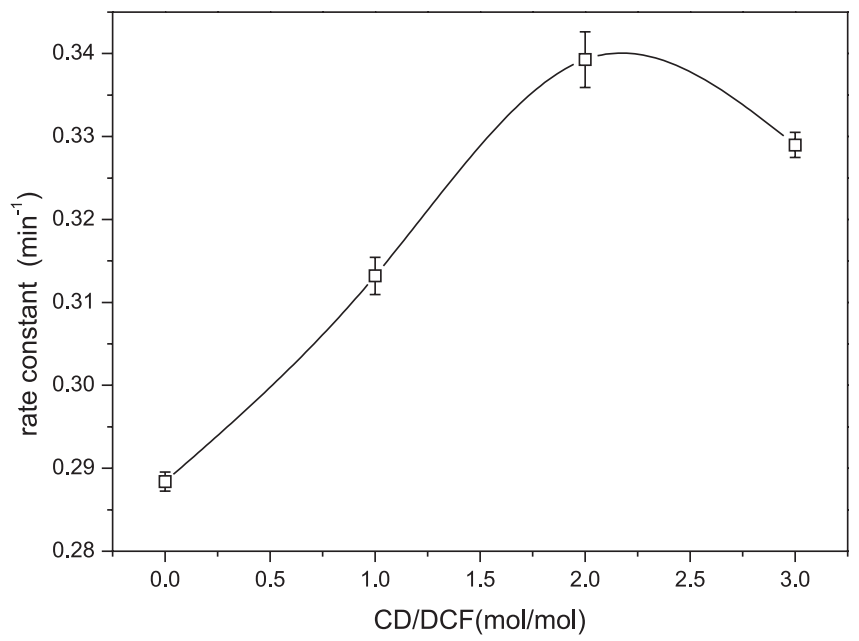

Figure 8 . The rate constant versus the mole ratio of $D C F$ to $\beta-C D$

The photodegradation of diclofenac with $\beta$-CDs under sun light irradiation were examined giving the results showed in Figure 9. The results show that photodegradation with $\beta$-CDs had higher efficiency at longer irradiation times compared to photodegradation without $\beta$-CDs. The results agree with those produced under $100 \mathrm{~W}$ mercury lamps.

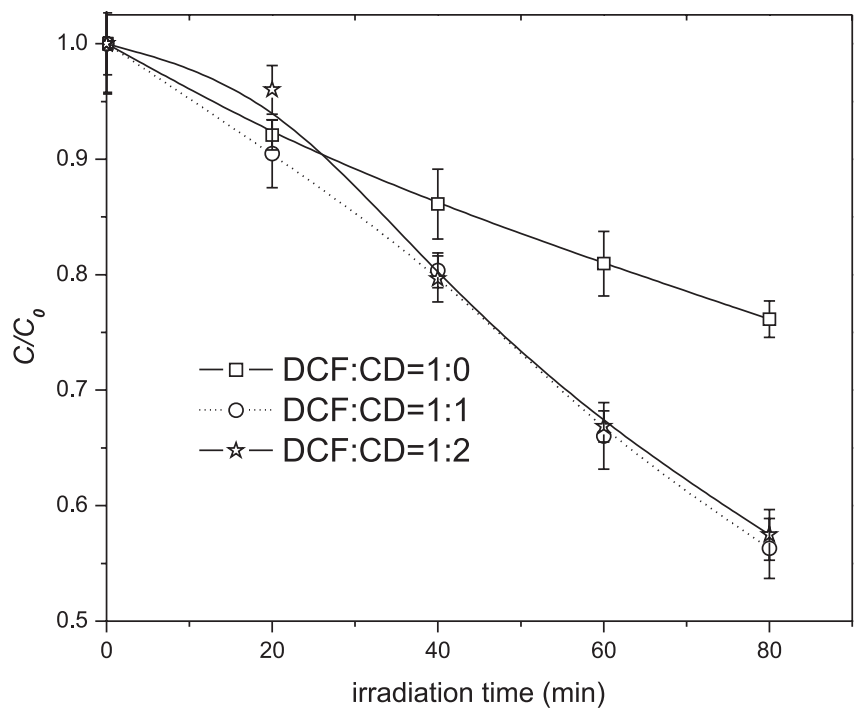

Figure 9. Effect of mole ratio of diclofenac to $\beta-C D$ under sun light

\section{CONCLUSION}

In this study, photo-induced transformation of diclofenac, and its liquid inclusion complexes with $\beta$-CDs, was studied in aqueous solution under different light sources. When initial DCF concentration was increased, the degradation process efficiency was low, resulting in longer half-life. The photodegradation of diclofenac followed pseudo-first-order kinetics under the different light sources. The rate constant of photodegradation under the lights, in descending order of magnitude, was Mercury lamp $>$ halogen lamp $>$ Xenon lamp $>$ Sunlight. UV-absorption spectra of diclofenac and phototransformation products were determined. One of the phototransformation products was identified. Irradiation of diclofenac in the presence of
$\beta$-CDs leads to a more rapid disappearance of the drug diclofenac under mercury lamp or sunlight, an effect which could be due to the behavior of complexed radical intermediates.

\section{SUPPLEMENTARY MATERIAL}

Available on http://quimicanova.sbq.org.br, in pdf file, with free access.

\section{ACKNOWLEDGMENTS}

This study is supported by Leading Academic Discipline Project of Shanghai Municipal Education Commission (Project Number: J51803), the National Natural Science Foundations of China (No. 50974087).

\section{REFERENCES}

1. Calza, P.; Sakkas, V. A.; Medana, C.; Baiocchi, C.; Dimou, A.; Pelizzetti, E.; Albanis, T.; Appl. Catal., B 2006, 67, 197.

2. Hartmann, J.; Bartels, P.; Mau, U.; Witter, M.; Tumpling, W. V.; Hofmann, J. ; Nietzschmann, E.; Chemosphere 2008, 70, 453.

3. Perez-Estrada, L. A.; Malato, S.; Gernjak, W.; Aguera, A.; Thurman, E. M.; Ferrer, I.; Fernandez-Alba, A. R.; Environ. Sci. Technol. 2005, 39, 8300.

4. Deng, A. P.; Himmelsbach, M.; Zhu, Q. Z.; Frey, S.; Sengl, M.; Buchberger, W.; Niessner, R.; Knopp, D.; Environ. Sci. Technol. 2003, 37,3422 .

5. Buser, H. R.;.Poiger, T.; Muller, M. D.; Environ. Sci. Technol. 1998, 32, 3449.

6. Andreozzi, R.; Raffaele, M.; Nicklas, P.; Chemosphere 2003, 50, 1319.

7. Packer, J. L.; Werner, J. J.; Latch, D. E.; McNeill, K.; Arnold, W. A.; Aquatic Sciences 2003, 65, 342.

8. Perez-Estrada, L. A.; Maldonado, M. I.; Gernjak, W.; Aguera, A.; Fernandez-Alba, A. R.; Ballesteros, M. M.; Malato, S.; Catal. Today 2005, 101, 219.

9. Garcia-Araya, J. F.; Beltran, F. J.; Aguinaco, A.; J. Chem. Technol. Biotechnol. 2010, 85, 798.

10. Deng, N. S.; Wang, G. H.; Qi, P. R.; Xue, X. F.; Wu, F.; Chemosphere 2007, 67, 762 .

11. Connors, K. A.; Chem. Rev. (Washington, DC, U. S.) 1997, 97, 1325.

12. Szejtli, J.; Chem. Rev. (Washington, DC, U. S.) 1998, 981743.

13. Deng, N. S.; Wang, G. H.; Xue, X. F.; Li, H. F.; Wu, F.; J. Mol. Catal. A: Chem. 2007, 276, 143.

14. Kamiya, M.; Nakamura, K.; Environ. Int. 1995, 21, 299.

15. Silva, A. P.; Gunaratne, H. Q. N.; Gunnlaugsson, T.; Chem. Rev. (Washington, DC, U. S.) 1997, 97, 1515.

16. Mora, M. J.; Longhi, M. R.; Granero, G. E.; Eur. J. Med. Chem. 2010 , 45, 4079.

17. Dias, M. M. R.; Raghavan, S. L.; Pellett, M. A.; Hadgraft, J.; Int. J. Pharm. 2003, 263, 173.

18. Miro, A.; Rondinone, A.; Nappi, A.; Ungaro, F.; Quaglia, F. L.; Rotonda, M. I.; Eur. J. Pharmaceutics and Biopharmaceutics 2009, 72, 76.

19. Kitano, H.; Endo, H.; Gemmei-ide, M.; Kyogoku, M.; J. Inclusion Phenom. Macrocyclic Chem. 2003, 47, 83.

20. Reddy, G. D.; Ramamurthy, V.; J. Org. Chem. 1987, 52, 3952.

21. Kamiya, M.; Nakamura, K.; Sasaki, C.; Chemosphere 1994, 28, 1961.

22. Eriksson, J.; Svanfelt, J.; Kronberg, L.; Photochem. Photobiol. 2010, 86, 528.

23. Valero, M.; Carrillo, C.; J. Photochem. Photobiol., B 2004, 74, 151.

24. Jimenez, M. C.; Miranda, M. A.; Tormos, R.; J. Photochem. Photobiol., A 1997, 104, 119. 


\section{PHOTOCHEMICAL CHARACTERISTICS OF DICLOFENAC AND ITS PHOTODEGRADATION OF INCLUSION COMPLEXES WITH $\beta$-CYCLODEXTRINS}

\section{Minmin Qin and Haifeng Yang}

School of Biology and Environmental Science, Shanghai Normal University, ShangHai 200234, PR China

Shengwen Chen*, Hongyong Xie and Jie Guan

School of Urban Development and Environment Engineering, Shanghai Second Polytechnic University, ShangHai 201209, PR China

Table 1S. Spectral energy distribution of mercury lamp

\begin{tabular}{lccccccccc}
\hline Wavelength $\mathrm{nm}$ & $265.2 \sim 265.5$ & 296.7 & $302.2 \sim 302.8$ & $312.6 \sim 313.2$ & $365.0 \sim 366.3$ & $404.5 \sim 407.8$ & 435.8 & 546.1 & $577.0 \sim 579.0$ \\
\hline Energy \% & 15.3 & 16.6 & 23.9 & 49.9 & 100.0 & 42.2 & 77.5 & 93.0 & 76.5 \\
\hline
\end{tabular}

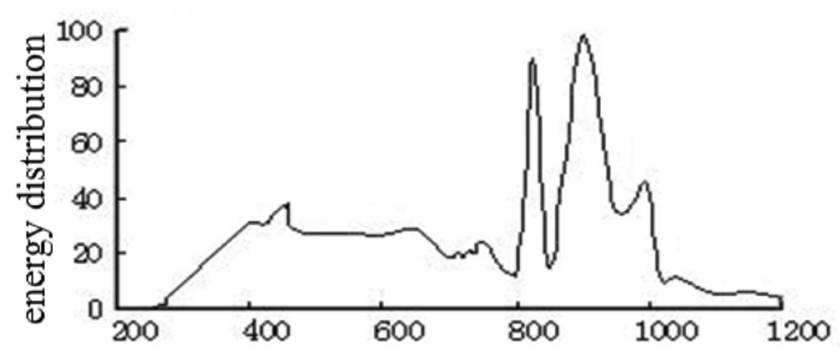

wavelength $\mathrm{nm}$

Figure 1S. Spectral energy distribution of xenon lamp

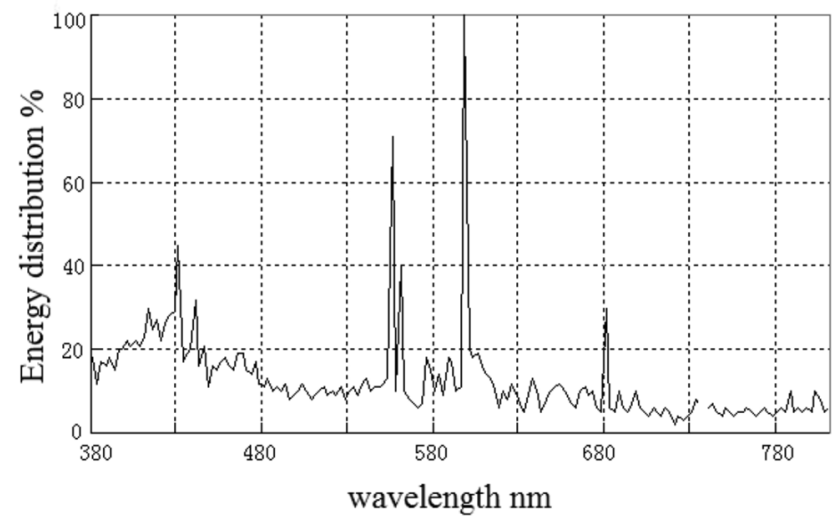

Figure 2S. Spectral energy distribution of halogen lamp

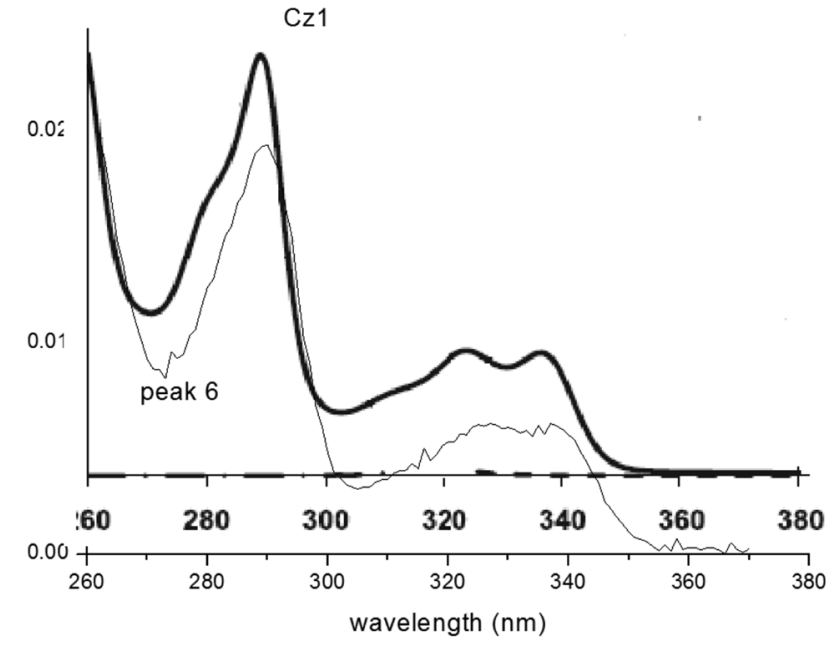

- Absorption spectrum of Cz1 was obtained from Figure 1 of ref. 22

Figure 3S. Comparison of UV spectra between peak 6 and $C z 1$ 